\title{
Prognostic Factors in Patients with Rhabdomyosarcoma Using Competing-Risks Analysis: A Study of Cases in the SEER Database
}

\author{
Didi Han, ${ }^{1,2}$ Chengzhuo Li, ${ }^{1,2}$ Xiang Li, ${ }^{3}$ Qiao Huang, ${ }^{4}$ Fengshuo Xu, ${ }^{1,2}$ Shuai Zheng, \\ Hui Wang, ${ }^{1,2}$ and Jun Lyu $\mathbb{D}^{1}$ \\ ${ }^{1}$ Department of Clinical Research, The First Affiliated Hospital of Jinan University, Guangzhou, \\ Guangdong Province 510630, China \\ ${ }^{2}$ School of Public Health, Xi'an Jiaotong University Health Science Center, Xi'an, Shaanxi Province 710061, China \\ ${ }^{3}$ School of Mechanical Engineering, Xi'an Jiaotong University, Xi'an 710049, China \\ ${ }^{4}$ Center for Evidence-Based and Translational Medicine, Zhongnan Hospital of Wuhan University, Wuhan 430071, China \\ ${ }^{5}$ School of Public Health, Shannxi University of Chinese Medicine, Xianyang, Shaanxi, China \\ Correspondence should be addressed to Jun Lyu; lyujun2020@jnu.edu.cn
}

Received 25 April 2020; Accepted 28 August 2020; Published 17 September 2020

Academic Editor: Muhammad Shahid

Copyright ( 2020 Didi Han et al. This is an open access article distributed under the Creative Commons Attribution License, which permits unrestricted use, distribution, and reproduction in any medium, provided the original work is properly cited.

\begin{abstract}
Background. Rhabdomyosarcoma (RMS) is a rare malignant soft-tissue sarcoma characterized by a poor outcome and unclear prognostic factors. This study applied a competing-risks analysis using data from the Surveillance, Epidemiology, and End Results (SEER) database to RMS patients, with the aim of identifying more accurate prognostic factors. Methods. Data of all patients with RMS during 1986-2015 were extracted from the SEER database. We used the competing-risks approach to calculate the cumulative incidence function (CIF) for death due to rhabdomyosarcoma (DTR) and death from other causes (DOC) at each time point. The Fine-Gray subdistribution proportional-hazards model was then applied in univariate and multivariate analyses to determine how the CIF differs between groups and to identify independent prognostic factors. The potential prognostic factors were analyzed using the competing-risks analysis methods in SAS and $\mathrm{R}$ statistical software. Results. This study included 3399 patients with RMS. The 5-year cumulative incidence rates of DTR and DOC after an RMS diagnosis were $39.9 \%$ and $8.7 \%$, respectively. The multivariate analysis indicated that age, year of diagnosis, race, primary site, historic stage, tumor size, histology subtype, and surgery status significantly affected the probability of DTR and were independent prognostic factors in patients with RMS. A nomogram model was constructed based on multivariate models for DTR and DOC. The performances of the two models were validated by calibration and discrimination, with C-index values of 0.758 and 0.670 , respectively. Conclusions. A prognostic nomogram model based on the competing-risks model has been established for predicting the probability of death in patients with RMS. This validated prognostic model may be useful when choosing treatment strategies and for predicting survival.
\end{abstract}

\section{Introduction}

Rhabdomyosarcoma (RMS) involves a rare malignant neoplasm of striated muscle. It constitutes $3 \%$ of all softtissue sarcomas in adults, while the estimated annual incidence in the US is 4.5 cases per 1 million children and adolescents $[1,2]$. RMS is characterized by a poor prognosis and unclear prognostic factors. The primary treatment of RMS includes chemotherapy, surgery, and radiation. The identification of prognostic factors for RMS may help with optimizing treatment protocols [3]. For all soft-tissue sarcomas, RMS accounts for $19 \%$ of such cases with adults and $45 \%$ of those in children [4]. Rhabdomyosarcoma is derived from primary mesenchymal cells, which presents as the skeletal muscle differentiation. There are two major histologic subtypes of RMS: embryonal (ERMS) for the younger patients and alveolar (ARMS) for older patients. The previous one typically arises in the head/neck and GU locations, 
while the latter one typically develops in the trunk and extremity locations [5]. There have been many reports on prognostic factors for RMS of the head, neck, limbs, and urogenital system [2, 3, 6-12]. Previous studies [13] have analyzed prognostic factors using the Kaplan-Meier method and the Cox proportional-hazards model, in which outcome events were classified into two categories: death or censored observation. Traditional survival analysis treats such competing risks by censoring, which will lead to an inaccurate survival function [14]. This is because the Kaplan-Meier method and the Cox method treat other competing events as censored, and the resulting estimates might be high or even inconsistent with the data, and this is also called competingrisks bias [15]. Therefore, competing-risks methods based on the subdistribution proportional hazards are recommended. However, to our knowledge, a competing-risks analysis and nomogram for RMS based on the Fine-Gray subdistribution proportional-hazards model have not been reported previously.

This study considered two types of failure event: death due to rhabdomyosarcoma (DTR) and death from other causes (DOC). We conducted a competing-risks analysis of patients with RMS using the Surveillance, Epidemiology, and End Results (SEER) database. We also constructed a simple competing-risks nomogram to evaluate the probabilities of DTR and DOC.

\section{Patients and Methods}

2.1. Study Patients. The study sample was derived from the SEER program, which collects demographic, diagnostic, and treatment information on all newly diagnosed cancer patients residing within specific geographic regions of the US. The SEER program collects information on incidence, prevalence, and survival, and its registry currently covers about $28 \%$ of the US population. The characteristics of the SEER population are comparable with those of the general US population.

The population included in this study comprised all patients who were diagnosed with RMS between 1986 and 2015, as identified using SEER ${ }^{*}$ Stat software (version 8.3.6.1). The study sample consisted of patients with the following ICD-O-3 (third revision of the International Classification of Diseases for Oncology) histology codes: $8900 / 0,8900 / 3,8901 / 3,8902 / 3,8903 / 0,8904 / 0,8905 / 0$, $8910 / 3,8912 / 3,8920 / 3,8921 / 3$, and $8991 / 3$. Only patients diagnosed with their first primary malignant tumor were included in this study. The following exclusion criteria were applied: (1) diagnosed at autopsy or by death certificate only, (2) no microscopic confirmation of the diagnosis, or (3) missing or incomplete information on survival, follow-up duration, or cause of death. After applying the exclusion criteria, the study population comprised 3399 patients with RMS. The flow chart for data selection is shown in Figure 1.

2.2. Defining Patient Characteristics. The following 14 variables related to each RMS case were selected from the SEER database: age, year of diagnosis, race, sex, marital
All patients with rhabdomyosarcoma diagnosed during 1986-2015 from 18 population-based cancer registries $(N=4145)$

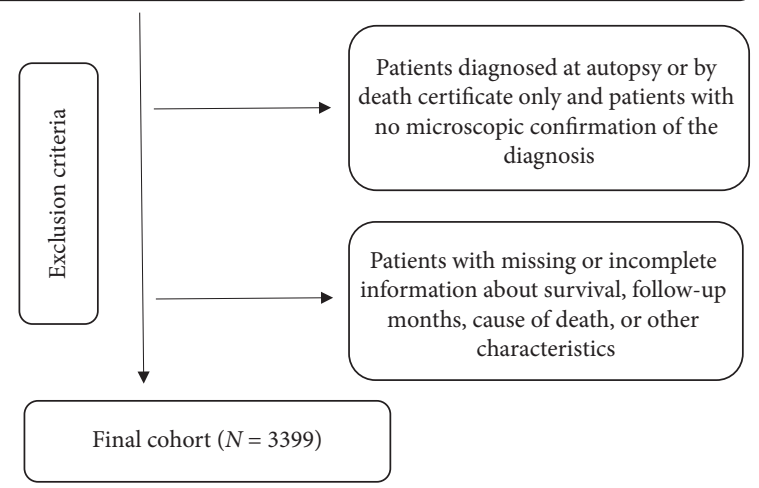

FIgURE 1: The inclusion and exclusion process of the study sample.

status, primary site, historic stage, tumor size, laterality, histology subtype (HS), radiation sequence with surgery (RS), surgery status, chemotherapy status, and radiotherapy status. Follow-up information and the cause of death were also extracted from the SEER database. Age was classified into $<19$ and $\geq 19$ years. The year of diagnosis was classified into 1986-1995, 1996-2005, and 2006-2015. Race was classified into white, black, and others. Sex was classified into male and female. Marital status was classified into married, unmarried, and divorced/separated/widowed (DSW). Tumor site was categorized into favorable, unfavorable, and unknown (missing), in accordance with the criteria used for staging pediatric tumors [16]. The head and neck (nonparameningeal), genitourinary (not bladder or prostate), and bile-duct regions were defined as favorable sites, while all other sites were regarded as unfavorable. We used the SEER "Historic Stage A" to classify the tumor stage into localized, regional, and distant. The tumor size was classified into $<5,5-10, \geq 10 \mathrm{~cm}$, and "other status." Laterality was classified into left, right, and "not a paired site." HS was classified into RMS, embryonal, alveolar, pleomorphic, and other. RS was classified into no radiation and/or cancer-directed surgery (NRS), radiation before and after surgery (WRS), and intraoperative radiation with radiation before or after surgery (IRS). The surgery status and radiotherapy status were defined as receiving or not receiving the corresponding therapy, while the chemotherapy status was classified into receiving and not/ unknown.

DTR was the primary endpoint. Consistent with the COD to site code, we classified the endpoint as alive, DTR or DOC. DTR were defined as cases in the SEER database where "SEER cause-specific death classification" was recorded as "Dead (attributable to this cancer $\mathrm{dx}$ )," while DOC were defined where this variable was recorded as "Death of other cause."

2.3. Statistical Analysis. The subdistribution proportionalhazards function is defined as the instantaneous possibility of occurrence of a given event in patients who have not 
experienced that type of event. The cumulative incidence function (CIF) describes the cumulative probability of the occurrence of a given event while accounting for competing events [17]. The Fine-Gray subdistribution proportional-hazards model was, then, applied in univariate and multivariate analyses to determine how the CIF differs between groups and to identify independent prognostic factors. Hazard ratios and their associated 95\% confidence intervals were calculated.

The model was internally validated. The C-index was used to measure the discrimination performance of the model and ranged from 0.5 (representing random chance) to 1.0 (representing a perfectly discriminating model). The model calibration-referring to the agreement between predicted and observed outcomes-was also checked. Furthermore, Fine-Gray proportional-hazards regression was performed to predict the 1-, 3-, and 5-year probabilities of the two competing death outcomes (i.e., DTR and DOC).

All statistical analyses were performed using SAS (version 9.4, SPSS, Chicago, IL, USA) and R (version 3.6.1; https://www.r-project.org/) statistical software. Several R packages were used to construct the model (survival, cmprsk, rms, mstate, survism, statmod, and eha), while the pec and risk regression packages were used to evaluate the model performance. All statistical tests were two-sided, with $P<0.05$ considered to be indicative of statistical significance.

\section{Results}

3.1. Patient Characteristics. The study included 3399 patients who met the inclusion criteria, of whom 1335 (39.28\%) were DTR patients and $344(10.12 \%)$ were DOC patients. The 3399 patients included $1976(58.1 \%)$ who were aged $<19$ years and $1489(43.8 \%)$ males. Most of the patients were white ( $n=2535,74.6 \%)$, unmarried (72.6\%), and had RMS at unfavorable sites (67.1\%). Based on the historic stage, 1127 (48.7\%) of patients had localized tumors, $24.5 \%$ had regional RMS, and $26.8 \%$ had distant metastasis. Tumors of size $5-10 \mathrm{~cm}$ were the most common $(n=1977,51.5 \%)$, and most of the tumors $(n=1890)$ were on the right side. Around $18.1 \%$ of patients were diagnosed with RMS: $38.9 \%$ with embryonal RMS, $24.8 \%$ with alveolar RMS, $10.4 \%$ with pleomorphic RMS, and 7.8\% with other RMS. NRS, WRS, and IRS were applied to $65.1 \%, 34.3 \%$, and $0.6 \%$ of the patients, respectively. Surgery, chemotherapy, and radiotherapy had been applied to $62.6 \%, 83.8 \%$, and $56.7 \%$ of the patients, respectively. The DTR patients comprised 341 married and 877 unmarried cases, while for the DOC group, the marital distribution was 137 (married) and 155 (unmarried), respectively. The proportions of those with primary-site surgery were $50.9 \%(n=680)$ and $67.7 \%(n=233)$ related to the DTR and DOC patients, respectively. For radiotherapy, $54.0 \%$ of DTR patients $(n=721)$ were treated with the radiation, while $40.4 \%(n=139)$ of DOC patients were given the same therapy. Chemotherapy was applied to $83.7 \%(n=1118)$ of the DTR patients and $51.5 \%(n=177)$ of the DOC patients. The demographic and tumor characteristics are listed in Table 1.
3.2. Univariate Analysis of the Prognosis of Rhabdomyosarcoma. The CIFs of DTR and DOC were $15.7 \%$ and $4.7 \%$, respectively, at 1 year, $35.3 \%$ and $7.4 \%$ at 3 years, and $39.9 \%$ and $8.7 \%$ at 5 years. The 1-, 3-, and 5-year estimates of the cumulative incidence rates of DTR and DOC according to age, year of diagnosis, race, sex, marital status, primary site, historic stage, tumor size, laterality, HS, RS, surgery status, chemotherapy status, and radiotherapy status are presented in Table 2. The analysis of 14 variables by univariate Gray's test revealed the characteristics for an age $<19$ years, white race, unmarried status, favorable site, localized historic stage, a tumor size $<5 \mathrm{~cm}$, and left laterality. The embryonal RMS was associated with a lower probability of DTR, while in NRS, no surgery, chemotherapy, and radiotherapy were associated with a higher probability of DTR. The historic stage and surgery status were not significantly related to the cumulative incidence of DTR. Age, year, race, sex, marital, site, historic stage, tumor size, laterality, HS, RS, chemotherapy, and radiotherapy significantly refer to the cumulative incidence of competing mortality. The CIF curves of DTR are shown in Figures 2(a)-2(j).

\subsection{Multivariate Analysis of the Prognosis of} Rhabdomyosarcoma. Table 3 presents the results of a multivariate analysis, which was performed by a Fine-Gray subdistribution proportional-hazards model. After adjusting for the variables that were significant in the univariate analysis by the CIF, the multivariate analysis found that age, race, primary site, historic stage, tumor size, HS, and surgery status could significantly affect DTR in patients with RMS. The probability of DTR was higher in RMS patients with an advanced age $(\mathrm{sdHR}=1.915, P<0.001)$, unfavorable site ( $\operatorname{sdHR}=1.241, P=0.011)$, and larger tumor $(\mathrm{sdHR}=1.447$, $P<0.001)$. Patients who belong to white race ( $\mathrm{sdHR}=0.837$, $P=0.042)$, localized historic stage $(\mathrm{sdHR}=0.294$, $P<0.001$ ), embryonal RMS (sdHR $=0.617, P<0.001$ ), and received surgery (sdHR $=0.720, P=0.002)$ owned a lower probability of DTR. However, the tumor-related factors of the year of diagnosis, race, sex, primary site, historic stage, tumor size, laterality, HS, RS, surgery status, and radiotherapy status were no longer associated with DOC. Instead, only age, marital status, and chemotherapy status were significantly associated with DOC, advanced age and DSW. In addition, no chemotherapy involves a higher risk of DOC.

3.4. Construction and Validation of the Nomogram. The nomogram that we developed based on the subdistribution proportional-hazards model is shown in Figure 3. All of the independent predictors of DTR and DOC in the entire study population were included in the predictive nomogram established for the 1-, 3-, and 5-year probabilities of DTR and DOC in the training cohort. The discrimination performance of the Fine-Gray model was evaluated based on the C-index, whose values for the 1-, 3-, and 5-year probabilities of DTR for the nomogram were $0.758,0.714$, and 0.707 in the derivation cohort and $0.769,0.739$, and 0.735 in the validation cohort, respectively. The related values of the DOC nomogram were $0.670,0.620$, and 0.609 in the 
TABLE 1: Baseline characteristics of patients with rhabdomyosarcoma.

\begin{tabular}{|c|c|c|c|c|}
\hline Variables & Classification & $N$ & Cause-specific death (\%) & Death due to other causes (\%) \\
\hline Total & & 3399 & 1335 & 344 \\
\hline \multirow{3}{*}{ Age } & $<19$ & $1976(58.1)$ & $629(47.1)$ & $57(16.6)$ \\
\hline & $\geq 19$ & $1423(41.9)$ & $706(52.9)$ & $287(83.4)$ \\
\hline & $1986-1995$ & $396(11.7)$ & $168(12.6)$ & $38(11.0)$ \\
\hline \multirow[t]{3}{*}{ Year } & 1996-2005 & $1250(36.8)$ & $527(39.5)$ & $130(37.8)$ \\
\hline & $2006-2015$ & $1753(51.5)$ & $640(47.9)$ & $176(51.2)$ \\
\hline & White & $2535(74.6)$ & $961(72.0)$ & $276(80.3)$ \\
\hline \multirow[t]{2}{*}{ Race } & Black & $580(17.1)$ & $249(18.7)$ & $51(14.8)$ \\
\hline & Other & $284(8.3)$ & $125(9.3)$ & $17(4.9)$ \\
\hline \multirow{3}{*}{ Sex } & Male & $1489(43.8)$ & $591(44.3)$ & $170(49.4)$ \\
\hline & Female & $1910(56.2)$ & $744(55.7)$ & $174(50.6)$ \\
\hline & Married & $682(20.1)$ & $341(25.5)$ & $137(39.8)$ \\
\hline \multirow[t]{2}{*}{ Marital } & Unmarried & $2466(72.6)$ & $877(65.7)$ & $115(33.5)$ \\
\hline & DSW & $251(7.3)$ & $117(8.8)$ & $92(26.7)$ \\
\hline \multirow{3}{*}{ Site } & Favorable & $1118(32.9)$ & $350(26.2)$ & $102(29.7)$ \\
\hline & Unfavorable & $2281(67.1)$ & $985(73.8)$ & $242(70.3)$ \\
\hline & Localized & $1127(48.7)$ & $224(16.8)$ & $113(32.8)$ \\
\hline \multirow[t]{3}{*}{ Historic stage } & Regional & $1169(24.5)$ & $409(30.6)$ & $116(33.7)$ \\
\hline & Distant & $1103(26.8)$ & $702(52.6)$ & $115(33.5)$ \\
\hline & $<5$ & $868(25.1)$ & $199(14.9)$ & $73(21.2)$ \\
\hline \multirow{4}{*}{ Tumor size $(\mathrm{cm})$} & $5-10$ & $969(51.5)$ & $360(26.9)$ & $86(25.0)$ \\
\hline & $\geq 10$ & $748(23.4)$ & $388(29.1)$ & $94(27.3)$ \\
\hline & Other & $814(23.9)$ & $388(29.1)$ & $91(26.5)$ \\
\hline & Left & $776(22.8)$ & $270(20.2)$ & $75(21.8)$ \\
\hline \multirow[t]{4}{*}{ Laterality } & Right & $1890(55.6)$ & $257(19.3)$ & $62(18.0)$ \\
\hline & Not a paired site & $733(21.6)$ & $808(60.5)$ & $207(60.2)$ \\
\hline & RMS & $615(18.1)$ & $293(21.9)$ & $125(36.3)$ \\
\hline & Embryonal & $1321(38.9)$ & $354(26.5)$ & $52(15.1)$ \\
\hline \multirow[t]{4}{*}{$H S$} & Alveolar & $843(24.8)$ & $451(33.8)$ & $53(15.4)$ \\
\hline & Pleomorphic & $352(10.4)$ & $154(11.5)$ & $91(26.5)$ \\
\hline & Others & $268(7.8)$ & $83(6.2)$ & $23(6.7)$ \\
\hline & NRS & $2213(65.1)$ & $947(70.9)$ & $249(72.4)$ \\
\hline \multirow[t]{2}{*}{$R S$} & WRS & $1167(34.3)$ & $382(28.6)$ & $94(27.3)$ \\
\hline & IRS & $19(0.6)$ & $6(0.5)$ & $1(0.3)$ \\
\hline \multirow{2}{*}{ Surgery } & Yes & $2127(62.6)$ & $680(50.9)$ & $233(67.7)$ \\
\hline & No/unknown & $1272(37.4)$ & $655(49.1)$ & $111(32.3)$ \\
\hline \multirow{2}{*}{ Chemotherapy } & Yes & $1926(56.7)$ & $1118(83.7)$ & $177(51.5)$ \\
\hline & None/unknown & $1473(43.3)$ & $217(16.3)$ & $167(48.5)$ \\
\hline \multirow{2}{*}{ Radiotherapy } & Yes & $2847(83.8)$ & $721(54.0)$ & $139(40.4)$ \\
\hline & No/unknown & $552(16.2)$ & $614(46.0)$ & $205(59.6)$ \\
\hline
\end{tabular}

DSW: divorced and separated and widowed; RMS: rhabdomyosarcoma; HS: histology subtype; RS: radiation sequence with surgery; NRS: no radiation and/or cancer-directed surgery; WRS: radiation prior to surgery, radiation after surgery, and radiation before and after surgery.

derivation cohort and $0.643,0.608$, and 0.601 in the validation one, respectively.

The internal calibration plots revealed a strong correlation between the predictions estimated by the nomogram and actual observations for both the training and validation cohorts. The calibration plots for 1-, 3-, and 5-year probabilities of DTR are shown in Figures 4 and 5. The dots in the plots fall close to the $45^{\circ}$ diagonal line, which suggests that the model was well calibrated for all predictions. The calibration plot showed good agreement between predicted and observed outcomes.

\section{Discussion}

RMS accounts for half of the soft-tissue sarcomas in children. Although it is the most common soft-tissue tumor, it is still rare, accounting for only $3-4 \%$ of pediatric cancers [18]. The present study evaluated DTR for 3399 patients with RMS who had been enrolled in the SEER database between 1986 and 2015; calculated the 1-, 3-, and 5-year CIFs; and constructed a nomogram to predict 1-, 3-, and 5-year probabilities of DTR and DOC. We found that 344 of 1699 patients were DOC, comprising $20 \%$ of the deaths, and this was taken as censored data based on the common method of survival analysis. The traditional method of analyzing specific causes of death may overestimate the cumulative incidence of each variable. Therefore, the Fine-Gray subdistribution proportional-hazards model was applied in this study to estimate the effects of covariates on the CIF and, therefore, identify the independent prognostic factors for RMS. A concise nomogram based on a competing-risks model was constructed to predict the probabilities of DTR and DOC. This prognostic tool will be useful for determining prognoses and guiding treatment selection. 
TABLE 2: Univariate analysis of prognostic factors in patients with rhabdomyosarcoma.

\begin{tabular}{|c|c|c|c|c|c|c|c|c|c|}
\hline \multirow[t]{2}{*}{ Variables } & \multirow[t]{2}{*}{ Classification } & \multicolumn{3}{|c|}{ Cancer-specific mortality (\%) } & \multirow[t]{2}{*}{$P$} & \multicolumn{3}{|c|}{$\begin{array}{c}\text { Non-cancer-specific mortality } \\
(\%)\end{array}$} & \multirow[t]{2}{*}{$P$} \\
\hline & & 1 year & 3 years & 5 years & & 1 year & 3 years & 5 years & \\
\hline Total & & 15.7 & 35.3 & 39.5 & $<0.001$ & 4.7 & 7.4 & 8.7 & $<0.001$ \\
\hline \multirow{3}{*}{ Age } & $<19$ & 8.3 & 26.3 & 31.3 & $<0.001$ & 0.5 & 1.4 & 2.0 & $<0.001$ \\
\hline & $\geq 19$ & 26.1 & 47.9 & 51.1 & & 10.7 & 16.0 & 18.2 & \\
\hline & 1986-1995 & 16.4 & 34.9 & 39.7 & 0.967 & 2.5 & 4.8 & 5.8 & 0.001 \\
\hline \multirow[t]{3}{*}{ Year } & $1996-2005$ & 15.7 & 35.8 & 39.6 & & 4.7 & 6.6 & 7.4 & \\
\hline & $2006-2015$ & 15.6 & 34.9 & 39.4 & & 5.2 & 8.8 & 10.5 & \\
\hline & White & 15.1 & 33.8 & 37.9 & 0.022 & 5.0 & 8.0 & 9.2 & 0.022 \\
\hline \multirow[t]{2}{*}{ Race } & Black & 17.7 & 40.0 & 43.3 & & 4.7 & 6.7 & 8.0 & \\
\hline & Other & 17.3 & 38.3 & 45.5 & & 2.8 & 4.8 & 5.7 & \\
\hline \multirow{3}{*}{ Sex } & Male & 17.1 & 35.9 & 40.6 & 0.269 & 6.3 & 9.2 & 10.3 & 0.011 \\
\hline & Female & 14.6 & 34.7 & 38.6 & & 3.5 & 6.1 & 7.4 & \\
\hline & Married & 27.1 & 48.1 & 51.1 & $<0.001$ & 10.3 & 16.8 & 19.3 & $<0.001$ \\
\hline \multirow[t]{2}{*}{ Marital } & Unmarried & 11.0 & 30.6 & 35.5 & & 1.5 & 2.7 & 3.5 & \\
\hline & DSW & 31.7 & 46.8 & 47.7 & & 21.6 & 29.3 & 31.8 & \\
\hline \multirow{3}{*}{ Site } & Favorable & 12.9 & 28.7 & 32.0 & $<0.001$ & 4.0 & 6.4 & 7.1 & $<0.001$ \\
\hline & Unfavorable & 17.1 & 38.5 & 43.2 & & 5.1 & 7.9 & 9.5 & \\
\hline & Localized & 5.8 & 16.1 & 19.1 & 0.000 & 2.8 & 5.8 & 7.9 & 0.841 \\
\hline \multirow[t]{3}{*}{ Historic stage } & Regional & 11.8 & 30.1 & 34.7 & & 4.7 & 7.0 & 8.1 & \\
\hline & Distant & 30.0 & 60.0 & 65.2 & & 6.7 & 9.6 & 10.1 & \\
\hline & $<5$ & 6.6 & 19.7 & 22.4 & 0.000 & 2.1 & 4.9 & 6.5 & 0.013 \\
\hline \multirow{4}{*}{ Tumor size } & $5-10$ & 11.6 & 33.2 & 38.0 & & 4.1 & 6.2 & 7.5 & \\
\hline & $\geq 10$ & 26.2 & 47.4 & 51.9 & & 7.4 & 10.3 & 11.6 & \\
\hline & Other & 20.8 & 43.0 & 47.7 & & 5.9 & 9.0 & 9.7 & \\
\hline & Not a paired site & 14.6 & 34.6 & 38.1 & $<0.001$ & 4.5 & 7.2 & 8.2 & $<0.001$ \\
\hline \multirow[t]{4}{*}{ Laterality } & Left & 12.9 & 29.1 & 34.3 & & 3.4 & 5.5 & 7.2 & \\
\hline & Right & 17.4 & 38.0 & 42.1 & & 5.4 & 8.3 & 9.5 & \\
\hline & RMS & 26.7 & 44.8 & 48.4 & 0.000 & 10.3 & 15.5 & 18.2 & 0.000 \\
\hline & Embryonal & 9.4 & 23.1 & 26.7 & & 1.5 & 2.3 & 2.5 & \\
\hline \multirow[t]{4}{*}{$H S$} & Alveolar & 14.7 & 47.0 & 53.3 & & 2.3 & 4.6 & 5.9 & \\
\hline & Pleomorphic & 26.2 & 43.4 & 45.4 & & 14.0 & 20.4 & 23.1 & \\
\hline & Other & 11.3 & 24.3 & 29.0 & & 3.8 & 6.6 & 7.5 & \\
\hline & NRS & 20.2 & 39.7 & 43.3 & $<0.001$ & 5.9 & 8.9 & 10.0 & $<0.001$ \\
\hline \multirow[t]{2}{*}{$R S$} & WRS & 7.5 & 26.9 & 32.3 & & 2.6 & 4.8 & 6.3 & \\
\hline & IRS & 5.6 & 27.8 & 33.3 & & 0.0 & 0.0 & 0.0 & \\
\hline \multirow{2}{*}{ Surgery } & Yes & 11.7 & 27.2 & 31.6 & 0.000 & 4.5 & 7.4 & 9.0 & 0.053 \\
\hline & No/unknown & 22.5 & 48.7 & 52.7 & & 5.1 & 7.6 & 8.2 & \\
\hline \multirow{2}{*}{ Chemotherapy } & Yes & 14.2 & 34.8 & 39.4 & 0.172 & 2.4 & 4.5 & 5.4 & 0.000 \\
\hline & No/unknown & 23.7 & 37.6 & 39.9 & & 16.8 & 22.5 & 26.0 & \\
\hline \multirow{2}{*}{ Radiotherapy } & Yes & 10.5 & 32.6 & 37.6 & $<0.001$ & 2.7 & 4.8 & 5.7 & $<0.001$ \\
\hline & None/unknown & 22.6 & 38.7 & 41.9 & & 7.5 & 10.9 & 12.5 & \\
\hline
\end{tabular}

DSW: divorced and separated and widowed; RMS: rhabdomyosarcoma; HS: histology subtype; RS: radiation sequence with surgery; NRS: no radiation and/or cancer-directed surgery; WRS: radiation prior to surgery, radiation after surgery, and radiation before and after surgery; IRS: intraoperative radiation and intraoperative rad with other rad before/after surgery.

Previous studies of the prognosis status of RMS have widely used Kaplan-Meier estimates of survival curves and Cox regression models to describe survival trends and identify important prognostic factors $[19,20]$. Competing-risks nomograms have been established for other tumors, such as breast cancer, prostate cancer, thyroid cancer, kidney cancer, sarcoma, melanoma, and head and neck squamous cell carcinoma [21-27]. To our knowledge, the present study is the first to construct a nomogram for patients with RMS based on the Fine-Gray subdistribution proportional-hazards model. The model was found to perform well, and the predictive tool is also easy to use because the variables incorporated in the model can be obtained from clinical investigations.
Our univariate CIF analysis showed that the 5-year mortality probabilities for DTR and DOC were $39.5 \%$ and $8.7 \%$, respectively. This study is the first to conduct a risk analysis of RMS patients using a cumulative risk model in a competing-risks model and, thereby, identify more accurate prognostic factors. After adjusting for prognostic factors distinguished by the CIF, the Fine-Gray subdistribution proportional-hazards model indicated that the $P$ value was statistically significant for age, race, primary site, historic stage, tumor size, HS, and surgery status. The probability of DTR was higher in RMS patients with advanced age, black race, unfavorable site, distant historic stage, larger tumor, alveolar RMS, and no surgery. Similarly, advanced age, 

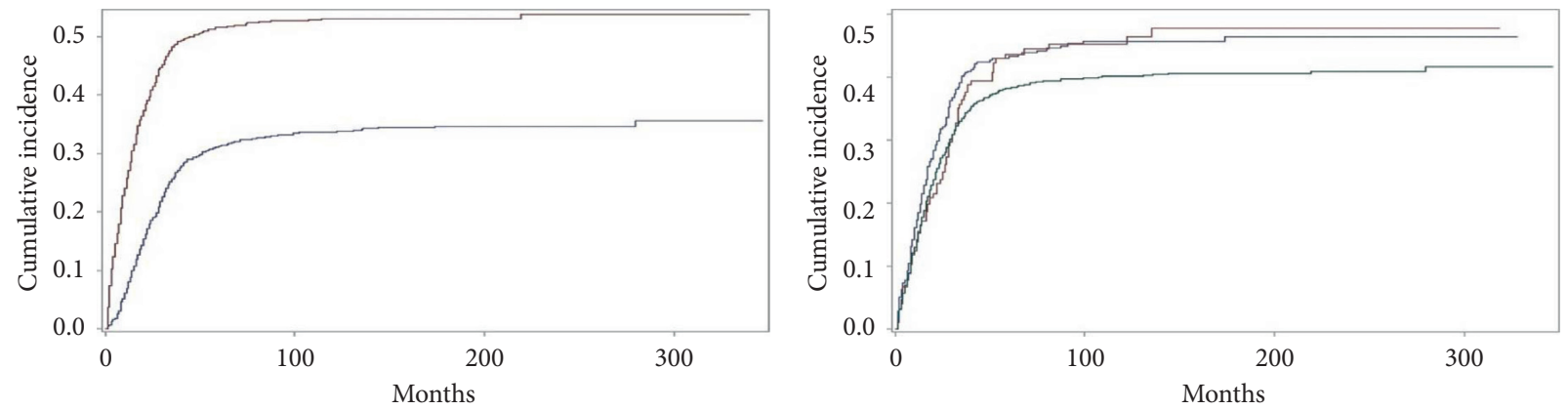

Age

$-<19$

Race

_ Black

- Other

— White

(a)

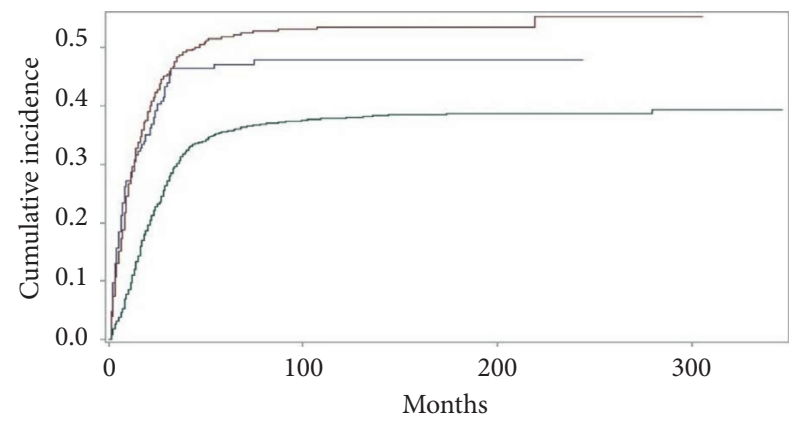

(b)

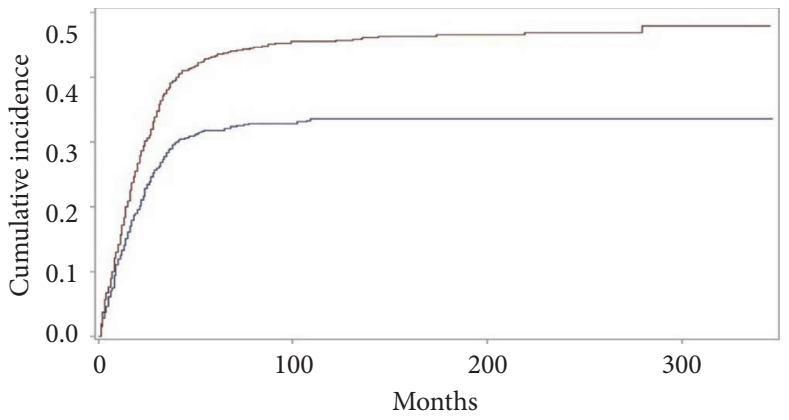

Marital

Site

— DSW

— Married

_ Favorable

_ Unfavorable

(c)

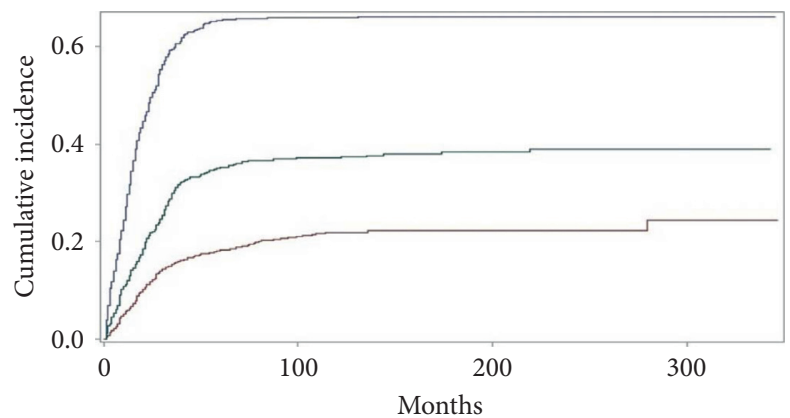

(d)

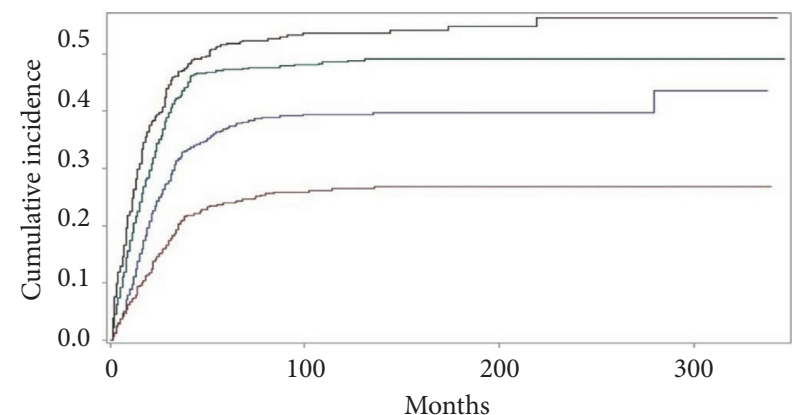

Historic

TS

- Distant
Localized
Regional

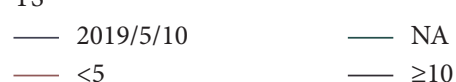

(e)

(f)

Figure 2: Continued. 

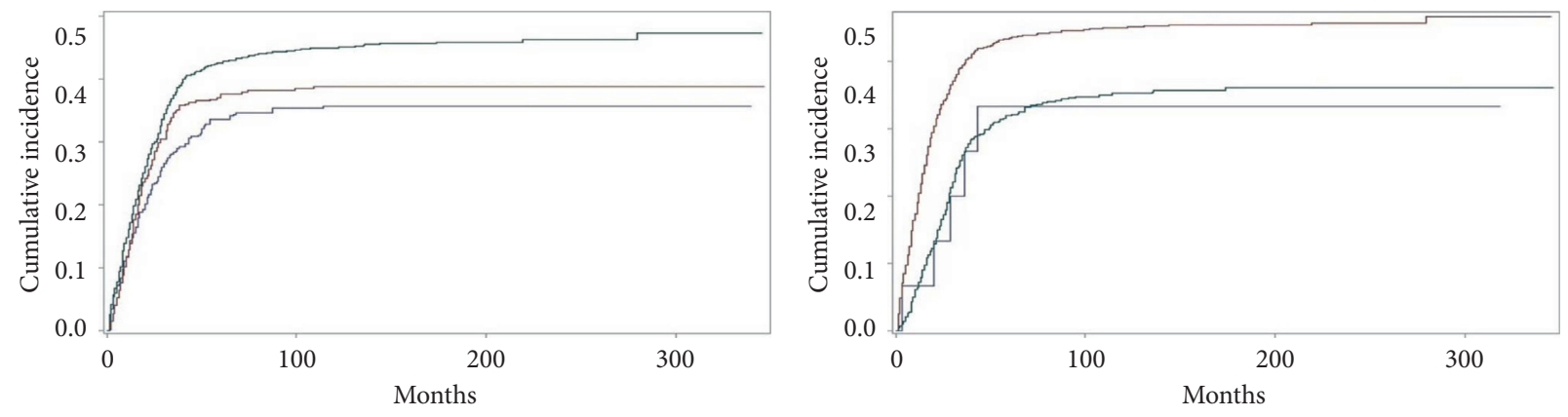

Laterality

— Left

_ Not a paired site

— Right

(g)

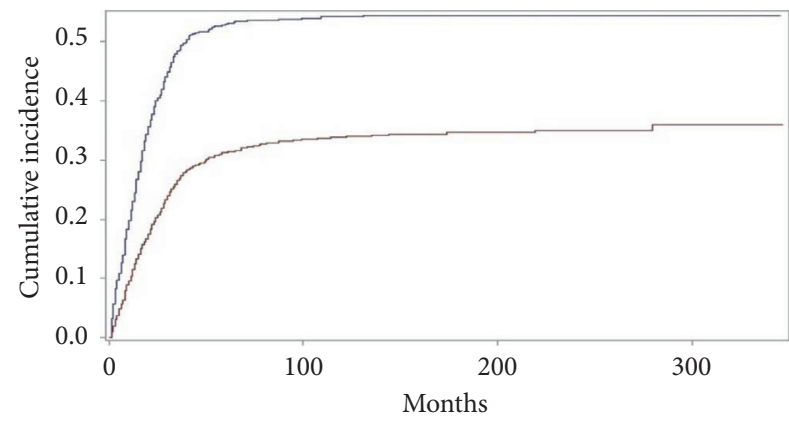

RS

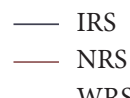

— WRS

(h)

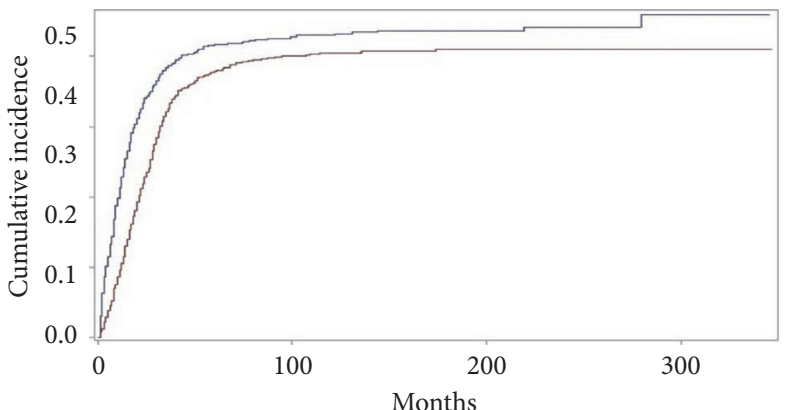

Surgey

Radiotherapy

_ None/unknown

— Yes

(i)

(j)

Figure 2: Cumulative incidence curves of cause-specific death according to patient characteristics (a-j).

DSW, and no chemotherapy increased the probability of DOC. We, then, utilized the independent predictive factors to create a monogram for the 1-, 3-, and 5-year probabilities of DTR and DOC. The C-index values and the calibration curves indicated that the model performed well in both the training and validation cohort.

Variations in survival rates with the age at diagnosis have been observed in many studies $[2,6,10,28]$. Sultan et al. [16] studied 2600 patients with a diagnosis of RMS and found that the outcomes were consistently worse for adults than for children regardless of the clinical characteristics. Our results were also similar to the previous research [2]; in that, older patients had a higher risk of DTR. In a multivariate analysis, a diagnosis during 1986-1995 was a significant independent predictor of a poor prognosis, which is consistent with previous research. A previous study [10] of survival data divided into decades found a similar trend, with a significantly worse 5 -year survival rate during the 1970s (46\%) than during the 1980 s and 1990 s (60\% and $64 \%$, respectively). Our analyses of racial differences in RMS incidence and survival were exploratory only due to the smallness of the samples in many of the compared categories.
The tumor primary site was classified into favorable and unfavorable based on criteria used for staging pediatric tumors. Our multivariate analysis results for DTR revealed significant differences. Other studies have also found that the survival rate of RMS is worse at unfavorable sites [16, 29], which is consistent with the present findings. Many authors have concluded that local and regional control is the most important factor for improving long-term survival [30-32]. The results obtained in our multivariate analysis of DTR also indicated that the postoperative survival was better for localized historic stage and regional RMS than for distant RMS, which is consistent with previous findings of distant RMS having an unfavorable prognosis. For smaller tumors with no evidence of metastasis, surgical extirpation alone might be the definitive treatment [33-35]. Moreover, Unsal et al. and Dantonello et al. reported that RMS with tumor size is a risk factor for poor survival $[6,36]$. This is consistent with our study finding that a larger tumor was an adverse prognostic factor in DTR patients.

In our study, the survival was better in embryonal than alveolar RMS patients, with 5-year mortality rates of $26.7 \%$ and $53.3 \%$, respectively. This finding of alveolar RMS being a significant independent predictor of a poor prognosis is 
TABLe 3: Proportional subdistribution hazards models for rhabdomyosarcoma.

\begin{tabular}{|c|c|c|c|c|c|c|c|}
\hline \multirow{2}{*}{ Variables } & \multirow{2}{*}{ Classification } & \multicolumn{2}{|r|}{ DTR } & \multirow{2}{*}{$P$} & \multicolumn{2}{|r|}{ DOC } & \multirow{2}{*}{$P$} \\
\hline & & Coefficient & sdHR (95\% CI) & & Coefficient & sdHR (95\% CI) & \\
\hline \multirow{3}{*}{ Age } & $<19$ & Reference & & & Reference & & \\
\hline & $\geq 19$ & 0.650 & $1.915(1.586-2.314)$ & $<0.001$ & 1.091 & $2.978(1.879-4.720)$ & $<0.001$ \\
\hline & 1986-1995 & Reference & & & Reference & & \\
\hline \multirow[t]{3}{*}{ Year } & $1996-2005$ & -0.260 & $0.771(0.625-0.950)$ & 0.015 & -0.156 & $0.856(0.570-1.284)$ & 0.452 \\
\hline & $2006-2015$ & -0.430 & $0.650(0.527-0.803)$ & $<0.001$ & -0.170 & $0.844(0.572-1.244)$ & 0.391 \\
\hline & Black & Reference & & & Reference & & \\
\hline \multirow[t]{2}{*}{ Race } & White & -0.178 & $0.837(0.705-0.993)$ & 0.042 & 0.069 & $1.071(0.734-1.563)$ & 0.722 \\
\hline & Other & -0.138 & $0.871(0.663-1.145)$ & 0.322 & -0.704 & $0.495(0.232-1.056)$ & 0.069 \\
\hline \multirow{3}{*}{ Sex } & Female & Reference & & & Reference & & \\
\hline & Male & -0.046 & $0.955(0.832-1.097)$ & 0.517 & 0.184 & $1.202(0.918-1.573)$ & 0.180 \\
\hline & DSW & Reference & & & Reference & & \\
\hline \multirow[t]{2}{*}{ Marital status } & Married & 0.010 & $1.010(0.747-1.367)$ & 0.947 & -0.565 & $0.569(0.406-0.795)$ & 0.001 \\
\hline & Unmarried & -0.142 & $0.868(0.629-1.198)$ & 0.388 & -0.963 & $0.382(0.249-0.585)$ & $<0.001$ \\
\hline \multirow{3}{*}{ Site } & Favorable & Reference & & & Reference & & \\
\hline & Unfavorable & 0.216 & $1.241(1.050-1.467)$ & 0.011 & 0.313 & $1.367(0.996-1.877)$ & 0.053 \\
\hline & Distant & Reference & & & Reference & & \\
\hline \multirow[t]{3}{*}{ Historic stage } & Localized & -1.225 & $0.294(0.239-0.362)$ & $<0.001$ & -0.215 & $0.806(0.546-1.191)$ & 0.280 \\
\hline & Regional & -0.695 & $0.499(0.425-0.585)$ & $<0.001$ & -0.063 & $0.939(0.657-1.343)$ & 0.732 \\
\hline & $5-10$ & Reference & & & Reference & & \\
\hline \multirow{4}{*}{ Tumor size } & $<5$ & -0.170 & $0.843(0.681-1.044)$ & 0.118 & -0.189 & $0.827(0.561-1.221)$ & 0.340 \\
\hline & $\geq 10$ & 0.370 & $1.447(1.199-1.747)$ & $<0.001$ & -0.228 & $0.796(0.545-1.163)$ & 0.238 \\
\hline & Other & 0.074 & $1.077(0.894-1.298)$ & 0.435 & -0.112 & $0.894(0.612-1.306)$ & 0.563 \\
\hline & Left & Reference & & & Reference & & \\
\hline \multirow[t]{4}{*}{ Laterality } & Not a paired site & 0.067 & $1.070(0.865-1.323)$ & 0.535 & 0.202 & $1.223(0.811-1.846)$ & 0.337 \\
\hline & Right & 0.120 & $1.128(0.941-1.351)$ & 0.192 & 0.316 & $1.371(0.948-1.982)$ & 0.094 \\
\hline & Alveolar & Reference & & & Reference & & \\
\hline & RMS & -0.253 & $0.777(0.625-0.965)$ & 0.022 & 0.384 & $1.468(0.954-2.258)$ & 0.081 \\
\hline \multirow[t]{4}{*}{$H S$} & Embryonal & -0.483 & $0.617(0.517-0.736)$ & $<0.001$ & -0.375 & $0.687(0.418-1.130)$ & 0.139 \\
\hline & Pleomorphic & -0.208 & $0.813(0.606-1.090)$ & 0.166 & 0.266 & $1.305(0.794-2.145)$ & 0.294 \\
\hline & Other & -0.470 & $0.625(0.459-0.852)$ & 0.003 & 0.067 & $1.069(0.554-2.063)$ & 0.842 \\
\hline & IRS & Reference & & & Reference & & \\
\hline \multirow[t]{2}{*}{$R S$} & NRS & 0.447 & $1.564(0.586-4.176)$ & 0.372 & -0.012 & $0.988(0.157-6.205)$ & 0.989 \\
\hline & WRS & 0.419 & $1.520(0.584-3.954)$ & 0.391 & 0.803 & $1.255(0.211-7.457)$ & 0.803 \\
\hline \multirow{2}{*}{ Surgery } & No/unknown & Reference & & & Reference & & \\
\hline & Yes & -0.328 & $0.720(0.587-0.884)$ & 0.002 & -0.195 & $0.823(0.545-1.244)$ & 0.356 \\
\hline \multirow{2}{*}{ Chemotherapy } & No/unknown & Reference & & & Reference & & \\
\hline & Yes & 0.200 & $1.222(0.959-1.557)$ & 0.105 & -0.792 & $0.453(0.328-0.626)$ & $<0.001$ \\
\hline \multirow{2}{*}{ Radiotherapy } & None/unknown & Reference & & & Reference & & \\
\hline & Yes & -0.148 & $0.862(0.700-1.062)$ & 0.163 & -0.459 & $0.632(0.389-1.026)$ & 0.064 \\
\hline
\end{tabular}

DSW: divorced and separated and widowed; RMS: rhabdomyosarcoma; HS: histology subtype; RS: radiation sequence with surgery; NRS: no radiation and/or cancer-directed surgery; WRS: radiation prior to surgery, radiation after surgery, and radiation before and after surgery; IRS: intraoperative radiation and intraoperative rad with other rad before/after surgery.

consistent with previous research that found HS to be associated with survival [37]. In a previous study, Dasgupta et al. [5] reported that local surgery treatment is one of the key aspects and the main prognostic factor in treatment of RMS. Moreover, our multivariate analysis results for DTR show that surgery produced significant effects, which is consistent with previous results.

DOC means death due to causes other than RMS, including cardiovascular disease, respiratory disease, and diabetes mellitus. We found that advanced age, DSW, and no chemotherapy increased the probability of DOC. Older age is associated with significant declines in bodily functions and resulting in worse compensatory capabilities. Thus, advanced age is the predominant factor affecting DOC. Being married is associated with a comfortable, confident, and enjoyable emotional state, and married patients also receive social support from their family and have favorable family financial circumstances [38]. Multiagent chemotherapy is currently the indicated treatment for all patients with RMS. Neoadjuvant chemotherapy is also utilized to obtain cytoreduction of unresectable tumors and facilitate subsequent surgical excision [39]. Studies have shown that pediatric RMS has better sensitivity to chemotherapy than those diagnosed in adults [40].

The main strength of this study was that our nomogram can be used to quantify the probability of DTR after a diagnosis of RMS on an individual basis. To our knowledge, this is the first effort to construct a nomogram based on the Fine-Gray subdistribution proportional-hazards model for predicting DTR in RMS. This predictive tool is easy to use 


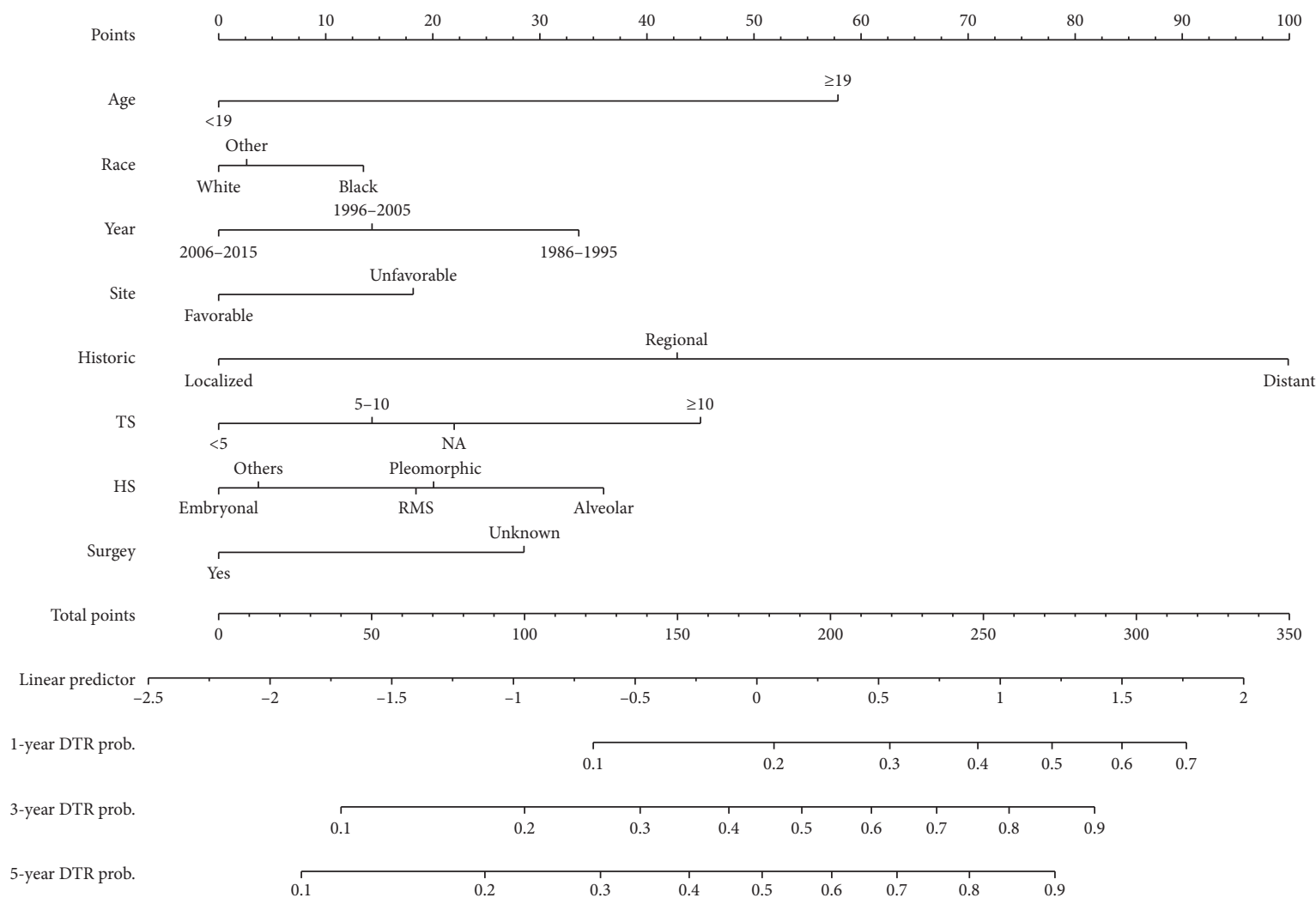

(a)

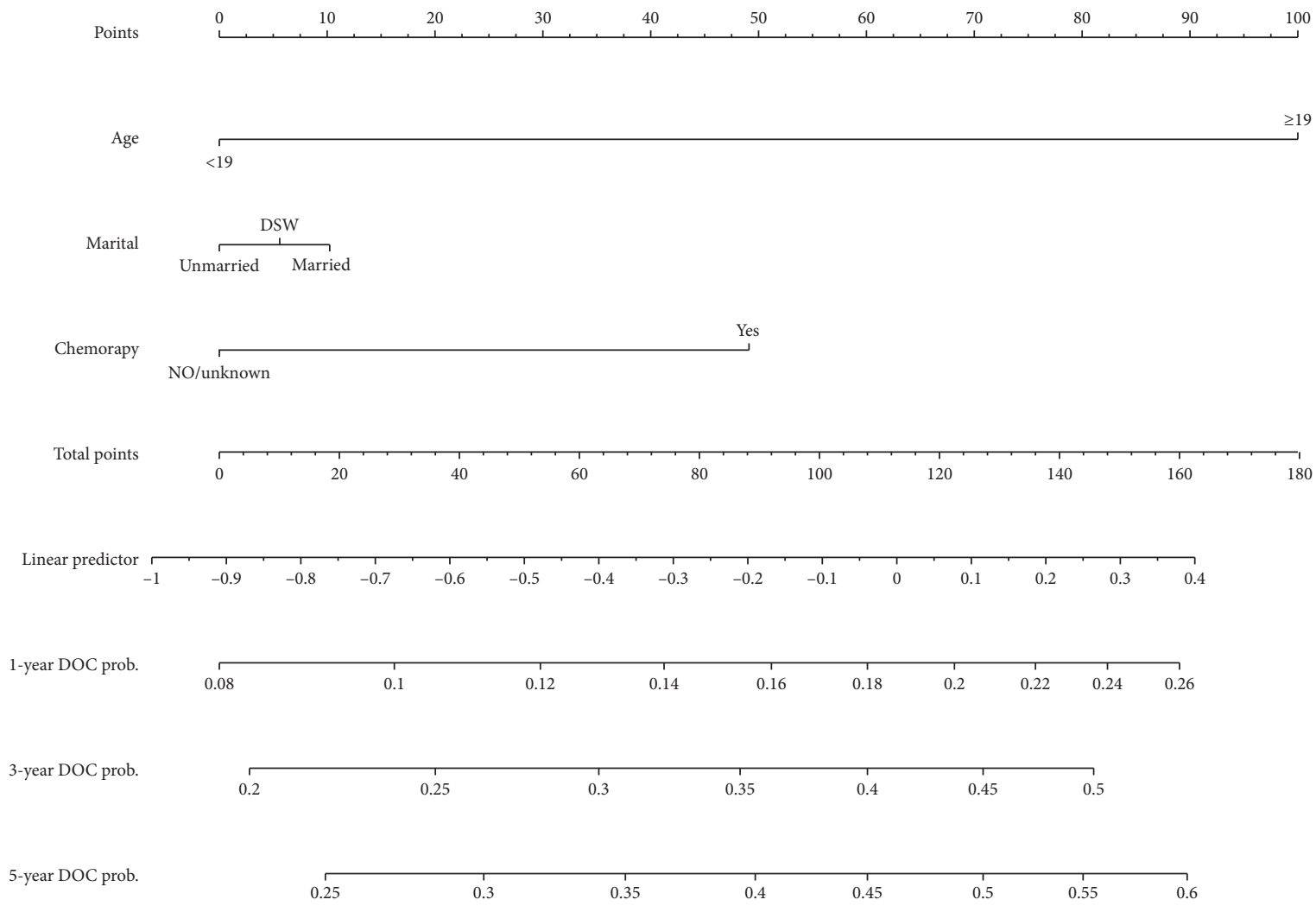

(b)

FIGURE 3: Competing-risks nomogram predicting 1-year, 3-year, and 5-year cumulative probabilities for DTR and DOC in patients with RMS. (a) RMS cancer-specific death; (b) other cause-specific death; TS, tumor size; HS, histology subtype; SEER, Surveillance, Epidemiology, and End Results. 


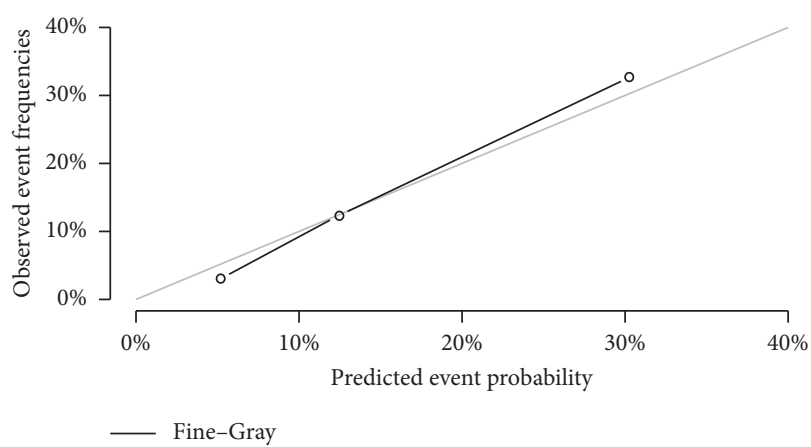

(a)

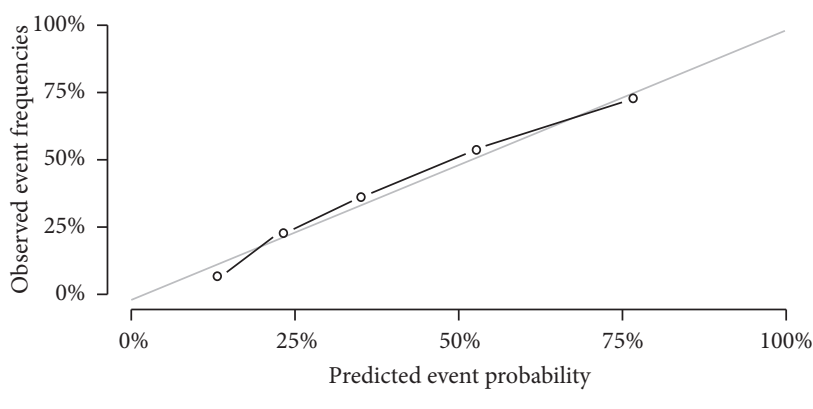

(c)

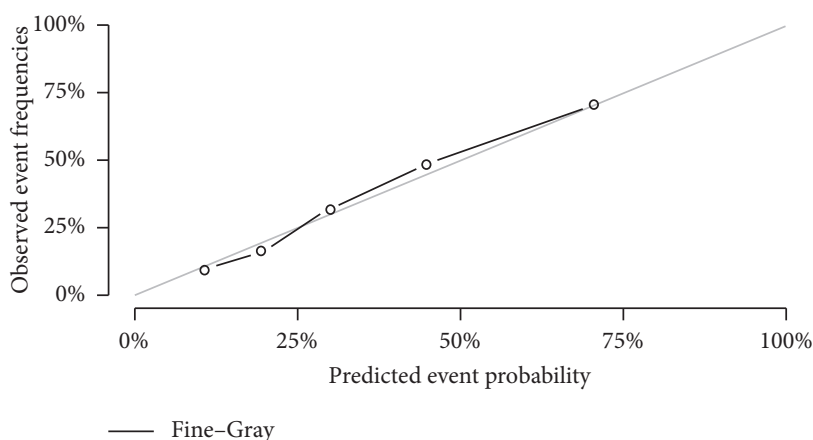

(e)

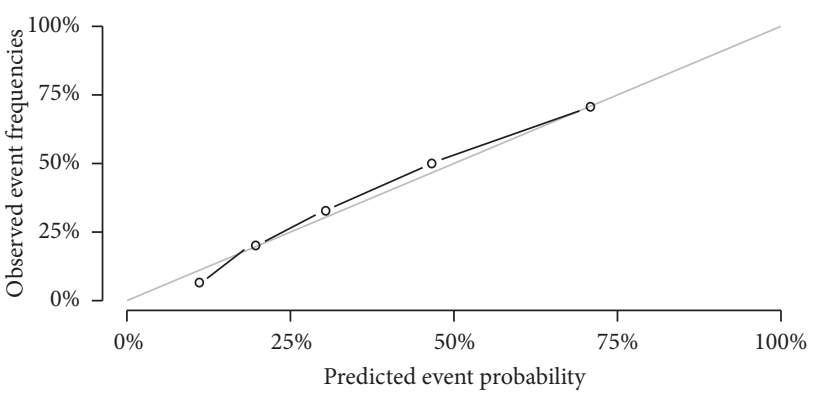

(b)

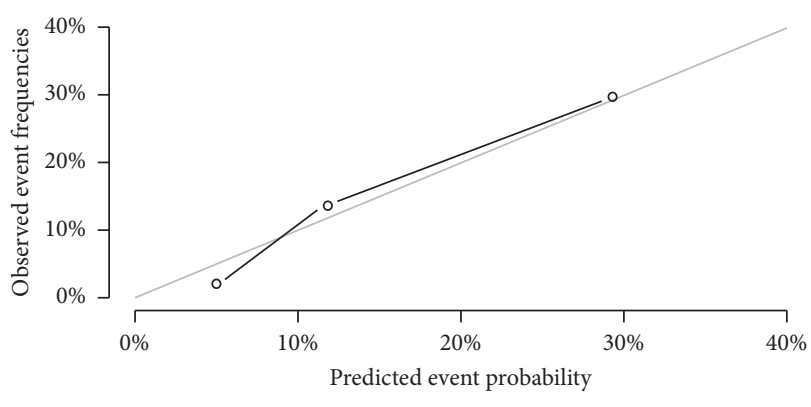

(d)

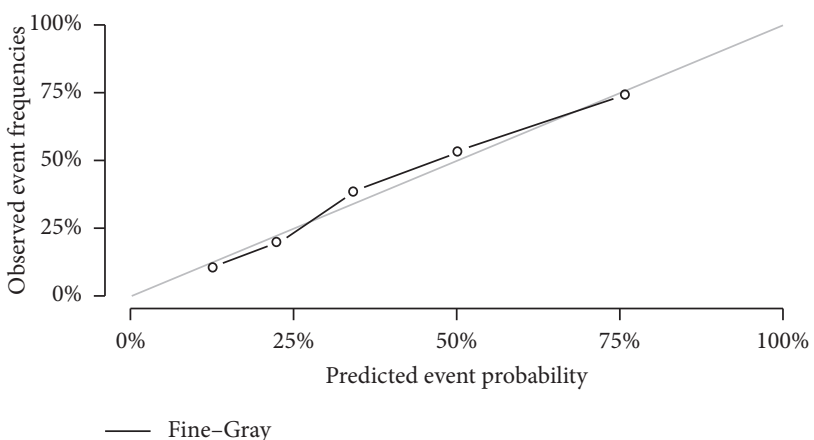

(f)

FIgURE 4: Calibration plots of the nomogram for 1-, 3-, and 5-year predicting RMS cause-specific mortality of the training set (a, b, c) and validation set $(d, e, f)$.

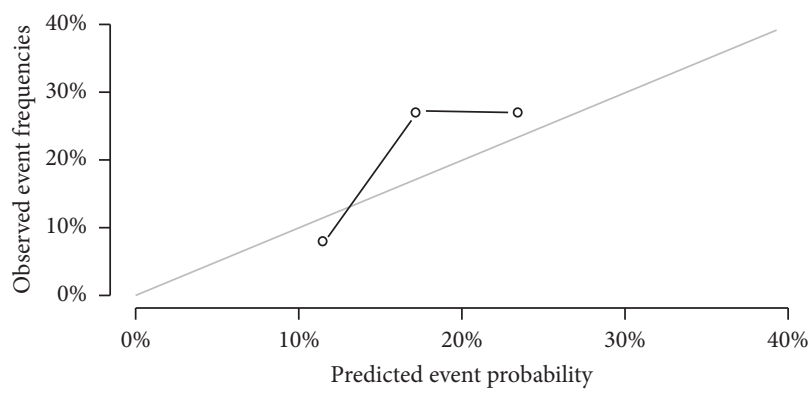

Fine-Gray

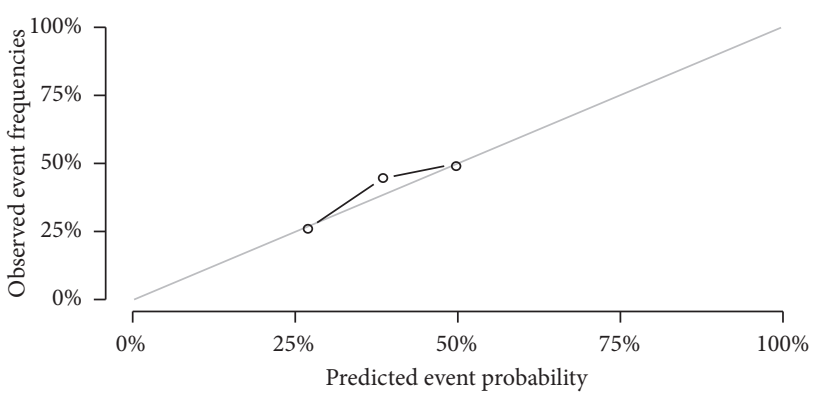

- Fine-Gray

(a)

(b)

Figure 5: Continued. 


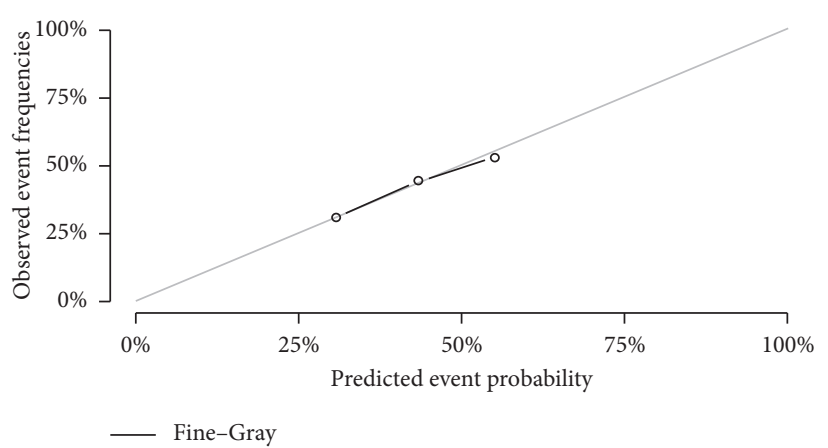

(c)

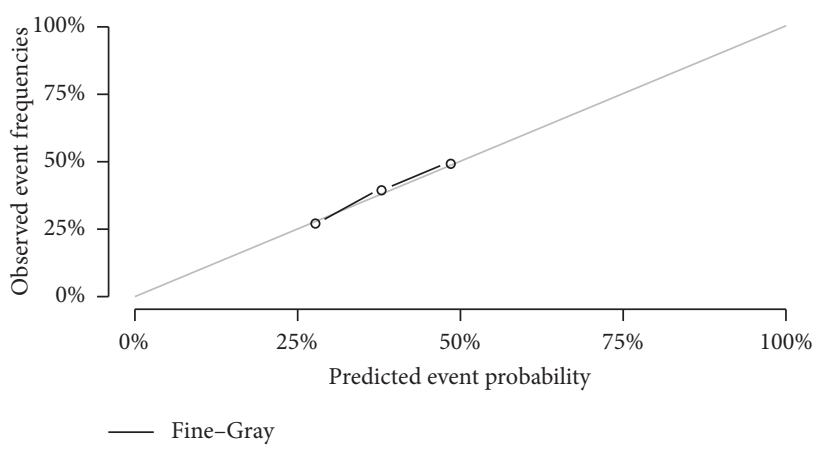

(e)

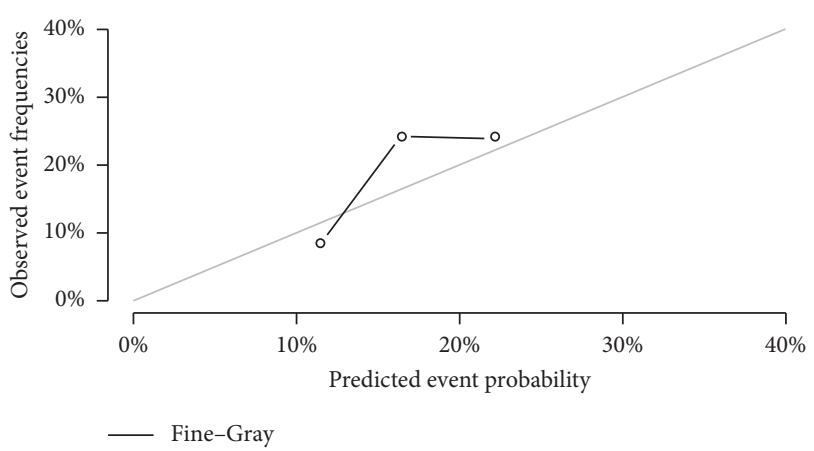

(d)

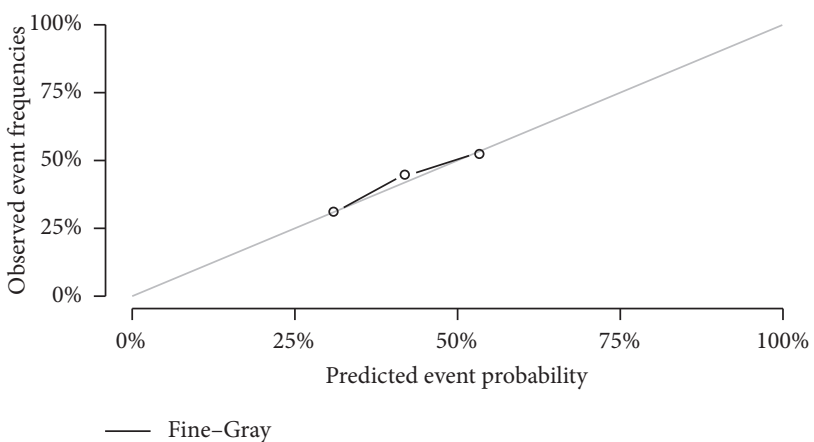

(f)

Figure 5: Calibration plots of the nomogram for 1-, 3-, and 5-year predicting other cause-specific mortality of the training set (a, b, c) and validation set $(d, e, f)$.

because the variables incorporated in the model can be obtained from clinical investigations. Therefore, based on combining clinical features and clinical information, this graphical predictive tool can be easily used by clinicians to make a prognosis judgment in a patient within seconds by drawing a few lines and without requiring any difficult calculations. Furthermore, SEER data are of high quality and are collected in a uniform manner with uniform data standards. The quality control of the SEER program ensures that there are relatively low rates of errors in the cancer registry.

This study was subject to a few limitations. First, there would have been differences from 1986 to 2015 in the types of surgery performed, in the experience of the surgeons, and in the grade classification of tumors, which may have interfered with the results. Second, the SEER database does not provide data on adjuvant therapy, comorbidities, or recurrence rates. Finally, despite being a user-friendly tool for helping clinicians to make clinical decisions, our nomogram did not include all possible prognostic factors and will not always provide an accurate prognosis for individual patients in clinical practice. Therefore, independent external validation is necessary to confirm the efficacy of the model.

\section{Conclusions}

In conclusion, we have evaluated the CIFs of DTR and DOC and performed a competing-risks analysis in patients with RMS using a large population-based sample from the SEER database. We also discovered independent predictive factors of DTR and DOC to build a nomogram. To our knowledge, we have produced the first competing-risks nomogram for calculating the 1-, 3-, and 5-year probabilities of DTR and DOC. Our nomogram performed relatively accurate and allows for objective and accurate selection of the patient population at high risk for rhabdomyosarcoma cause-specific mortality. We believe that these nomograms could be easily used by clinicians to predict prognosis and help determine a personalized treatment for DTR patients. However, further external validation is still needed.

\section{Data Availability}

The data used to support the findings of this study are available from the Surveillance, Epidemiology, and End Results (SEER) program (http://ssp://seerstat.imsweb.com: 2038-10025-Nov2019). All authors have access to data from this database. The interpretation and reporting of such data is the sole responsibility of the author.

\section{Disclosure}

The manuscript, or part of it, has neither been published (except in the form of an abstract or thesis) nor is currently under consideration for publication by any other journal.

\section{Conflicts of Interest}

The authors declare no conflicts of interest in this work. 


\section{Authors' Contributions}

D.H., C.L., and F.X participated in design, data collection, studies selection, data analysis/interpretation, and statistics and wrote the first draft; D.H., C.L., X.L., S.Z., F.X., and H.W. performed research and wrote the first draft; D.H., C.L., Q.H., X.L., S.Z., and F.X. collected and analyzed the data; all authors contributed to the design and interpretation of the study and to further drafts; all authors agree to be accountable for all aspects of the work; and L.J. is the guarantor.

\section{Acknowledgments}

The authors would like to thank the SEER program for providing open access to the database. This study was supported by the National Social Science Foundation of China (grant/award no. 16BGL183).

\section{References}

[1] S. W. Weiss, F. M. Enzinger, and J. R. Goldblum, "Enzinger and Weiss's soft tissue tumors," Human Pathology, vol. 39, no. 11, Article ID 1717, 2008.

[2] R. J. Lee, K. K. Lee, T. Lin, A. Arshi, S. A. Lee, and R. E. Christensen, "Rhabdomyosarcoma of the head and neck: impact of demographic and clinicopathologic factors on survival," Oral Surgery, Oral Medicine, Oral Pathology and Oral Radiology, vol. 124, no. 3, pp. 271-279, 2017.

[3] S. Ren, Z. Wang, X. Huang, L. Sun, J. Shao, and Z. Ye, "Prognostic factors for postoperative survival among patients with rhabdomyosarcoma of the limbs," Cancer Management and Research, vol. 10, pp. 4181-4189, 2018.

[4] I. Dziuba, P. Kurzawa, M. Dopierała, A. B. Larque, and D. Januszkiewicz-Lewandowska, "Rhabdomyosarcoma in children-current pathologic and molecular classification," Polish Journal of Pathology, vol. 69, no. 1, pp. 20-32, 2018.

[5] R. Dasgupta, J. Fuchs, and D. Rodeberg, "Rhabdomyosarcoma," Seminars in Pediatric Surgery, vol. 25, no. 5, pp. 276-283, 2016.

[6] A. A. Unsal, S. Y. Chung, A. B. Unsal, S. Baredes, and J. A. Eloy, "A population-based analysis of survival for sinonasal rhabdomyosarcoma," Otolaryngology-Head and Neck Surgery, vol. 157, no. 1, pp. 142-149, 2017.

[7] D. Nasioudis, M. Alevizakos, E. Chapman-Davis, S. S. Witkin, and K. Holcomb, "Rhabdomyosarcoma of the lower female genital tract: an analysis of 144 cases," Archives of Gynecology and Obstetrics, vol. 296, no. 2, pp. 327-334, 2017.

[8] C. H. Kirsch, M. Goodman, and N. Esiashvili, "Outcome of female pediatric patients diagnosed with genital tract rhabdomyosarcoma based on analysis of cases registered in SEER database between 1973 and 2006," American Journal of Clinical Oncology, vol. 37, no. 1, pp. 47-50, 2014.

[9] A. W. Wu, J. D. Suh, R. Metson, and M. B. Wang, "Prognostic factors in sinonasal sarcomas: analysis of the surveillance, epidemiology and end result database," The Laryngoscope, vol. 122, no. 10, pp. 2137-2142, 2012.

[10] J. H. Turner and J. D. Richmon, "Head and neck rhabdomyosarcoma: a critical analysis of population-based incidence and survival data," Otolaryngology-Head and Neck Surgery, vol. 145 , no. 6 , pp. 967-973, 2011.

[11] R. B. Raney, W. Lawrence, H. M. Maurer et al., "Rhabdomyosarcoma of the ear in childhood. A report from the intergroup rhabdomyosarcoma study-I," Cancer, vol. 51, no. 12, pp. 2356-2361, 1983.

[12] S. Sanghvi, P. Misra, N. R. Patel, E. Kalyoussef, S. Baredes, and J. A. Eloy, "Incidence trends and long-term survival analysis of sinonasal rhabdomyosarcoma," American Journal of Otolaryngology, vol. 34, no. 6, pp. 682-689, 2013.

[13] J. Zhong, Q. Zheng, T. An et al., "Nomogram to predict causespecific mortality in extensive-stage small cell lung cancer: a competing risk analysis," Thoracic Cancer, vol. 10, no. 9, pp. 1788-1797, 2019.

[14] H. A. David, "The theory of competing risks," Australian Journal Statistics, vol. 3, pp. 101-110, 1976.

[15] C. van Walraven and F. A. McAlister, "Competing risk bias was common in Kaplan-Meier risk estimates published in prominent medical journals," Journal of Clinical Epidemiology, vol. 69, pp. 170.e8-173.e8, 2016.

[16] I. Sultan, I. Qaddoumi, S. Yaser, C. Rodriguez-Galindo, and A. Ferrari, "Comparing adult and pediatric rhabdomyosarcoma in the surveillance, epidemiology and end results program, 1973 to 2005: an analysis of 2,600 patients," Journal of Clinical Oncology, vol. 27, no. 20, pp. 3391-3397, 2009.

[17] P. C. Austin and J. P. Fine, "Practical recommendations for reporting fine-gray model analyses for competing risk data," Statistics in Medicine, vol. 36, no. 27, pp. 4391-4400, 2017.

[18] K. M. Amer, J. E. Thomson, D. Congiusta et al., "Epidemiology, incidence, and survival of rhabdomyosarcoma subtypes: SEER and ICES database analysis," Journal of Orthopaedic Research, vol. 37, no. 10, pp. 2226-2230, 2019.

[19] L. Yang, T. Takimoto, and J. Fujimoto, "Prognostic model for predicting overall survival in children and adolescents with rhabdomyosarcoma," BMC Cancer, vol. 14, no. 1, p. 654, 2014.

[20] W. Shen, N. Sakamoto, and L. Yang, "Model to predict the survival benefit of radiation for patients with rhabdomyosarcoma after surgery: a population-based study," International Journal of Oncology, vol. 45, no. 2, pp. 549-557, 2014.

[21] W. Shen, N. Sakamoto, and L. Yang, "Melanoma-specific mortality and competing mortality in patients with nonmetastatic malignant melanoma: a population-based analysis," BMC Cancer, vol. 16, no. 1, p. 413, 2016.

[22] L. Yang, W. Shen, and N. Sakamoto, "Population-based study evaluating and predicting the probability of death resulting from thyroid cancer and other causes among patients with thyroid cancer," Journal of Clinical Oncology, vol. 31, no. 4, pp. 468-474, 2013.

[23] W. Shen, N. Sakamoto, and L. Yang, "Cancer-specific mortality and competing mortality in patients with head and neck squamous cell carcinoma: a competing risk analysis," Annals of Surgical Oncology, vol. 22, no. 1, pp. 264-271, 2015.

[24] A. J. Stephenson, M. W. Kattan, J. A. Eastham et al., "Prostate cancer-specific mortality after radical prostatectomy for patients treated in the prostate-specific antigen era," Journal of Clinical Oncology, vol. 27, no. 26, pp. 4300-4305, 2009.

[25] M. W. Kattan, G. Heller, and M. F. Brennan, "A competingrisks nomogram for sarcoma-specific death following local recurrence," Statistics in Medicine, vol. 22, no. 22, pp. 3515-3525, 2003.

[26] A. Kutikov, B. L. Egleston, Y.-N. Wong, and R. G. Uzzo, "Evaluating overall survival and competing risks of death in patients with localized renal cell carcinoma using a comprehensive nomogram," Journal of Clinical Oncology, vol. 28, no. 2, pp. 311-317, 2010.

[27] E. O. Hanrahan, A. M. Gonzalez-Angulo, S. H. Giordano et al., "Overall survival and cause-specific mortality of patients 
with stage T1a,bN0M0 breast carcinoma," Journal of Clinical Oncology, vol. 25, no. 31, pp. 4952-4960, 2007.

[28] J. A. Punyko, A. C. Mertens, K. S. Baker, K. K. Ness, L. L. Robison, and J. G. Gurney, "Long-term survival probabilities for childhood rhabdomyosarcoma. A populationbased evaluation," Cancer, vol. 103, no. 7, pp. 1475-1483, 2005.

[29] H. M. Maurer, T. Moon, M. Donaldson et al., "The intergroup rhabdomyosarcoma study: a preliminary report," Cancer, vol. 40, no. 5, pp. 2015-2026, 1977.

[30] B. K. Reilly, A. Kim, M. T. Peña et al., "Rhabdomyosarcoma of the head and neck in children: review and update," International Journal of Pediatric Otorhinolaryngology, vol. 79, no. 9, pp. 1477-1483, 2015.

[31] J. Radzikowska, W. Kukwa, A. Kukwa, A. Czarnecka, and A. Krzeski, "Review rhabdomyosarcoma of the head and neck in children,” Współczesna Onkologia, vol. 2, no. 2, pp. 98-107, 2015.

[32] C. F. Thompson, B. J. Kim, C. Lai et al., "Sinonasal rhabdomyosarcoma: prognostic factors and treatment outcomes," International Forum of Allergy \& Rhinology, vol. 3, no. 8, pp. 678-683, 2013.

[33] J. H. Simon, A. C. Paulino, R. B. Smith, and J. M. Buatti, "Prognostic factors in head and neck rhabdomyosarcoma," Head \& Neck, vol. 24, no. 5, pp. 468-473, 2002.

[34] M. B. Gillespie, D. T. Marshall, T. A. Day, A. O. Mitchell, D. R. White, and J. C. Barredo, "Pediatric rhabdomyosarcoma of the head and neck," Current Treatment Options in Oncology, vol. 7, no. 1, pp. 13-22, 2006.

[35] A. Ferrari, R. Miceli, C. Meazza et al., "Comparison of the prognostic value of assessing tumor diameter versus tumor volume at diagnosis or in response to initial chemotherapy in rhabdomyosarcoma," Journal of Clinical Oncology, vol. 28, no. 8, pp. 1322-1328, 2010.

[36] T. M. Dantonello, C. Int-Veen, P. Winkler et al., "Initial patient characteristics can predict pattern and risk of relapse in localized rhabdomyosarcoma," Journal of Clinical Oncology, vol. 26, no. 3, pp. 406-413, 2008.

[37] J. R. Kim, H. M. Yoon, K.-N. Koh, A. Y. Jung, Y. A. Cho, and J. S. Lee, "Rhabdomyosarcoma in children and adolescents: patterns and risk factors of distant metastasis," American Journal of Roentgenology, vol. 209, no. 2, pp. 409-416, 2017.

[38] W. Sun, Z. Qiu, W. Tan et al., "The influence of marital status on survival in patients with oral tongue squamous cell carcinoma," Oncotarget, vol. 8, no. 47, pp. 82092-82102, 2017.

[39] E. A. Perez, N. Kassira, M. C. Cheung, L. G. Koniaris, H. L. Neville, and J. E. Sola, "Rhabdomyosarcoma in children: a SEER population based study," Journal of Surgical Research, vol. 170, no. 2, pp. e243-e251, 2011.

[40] L. Zhu, Y. Sun, X. Wang et al., "Survival stratification in childhood rhabdomyosarcoma of the extremities: a derivation and validation study," Scientific Reports, vol. 10, no. 1, Article ID 5684, 2020. 